\title{
UM ESTUDO SOBRE A METODOLOGIA SEIS SIGMA
}

\section{A STUDY ON SIX SIGMA METHODOLOGY}

\author{
Jenifer Naiara de Gois - jenifer.gois@outlook.com \\ Faculdade de Tecnologia (Fatec) - Taquaritinga - SP - Brasil \\ Angelita Moutin Segoria Gasparotto - angelita.gasparotto@fatectq.edu.br \\ Faculdade de Tecnologia (Fatec) - Taquaritinga - SP - Brasil
}

DOI: 10.31510/infa.v17i2.969

Data de publicação: 18/12/2020

\begin{abstract}
RESUMO
O atual cenário está cada vez mais competitivo e os clientes mais exigentes. Pensando nisso iniciou-se a busca pela qualidade total e alta competitividade com o menor custo possível. O Seis Sigma, metodologia criada na década de 80 vem com esse objetivo, ele utiliza do embasamento estatístico para conquistar as metas estabelecidas. Tem como objetivo atingir a excelência na competitividade com a melhoria contínua dos processos, sendo uma estratégia gerencial para acelerar o aperfeiçoamento em processos, produtos e serviços. A expressão Sigma mensura a capacidade do processo a trabalhar isento de falhas. A contenção da oscilação no resultado cedido aos clientes em uma taxa de 3,4 imperfeições por milhão ou $99,99966 \%$ de perfeição define o Seis Sigma. O método se torna único e extremamente eficaz pela sua estruturação e foco na redução da variabilidade do processo. O estudo tem como objetivo apresentar os conceitos, definições e ferramentas do Seis Sigma e entender sua eficácia. A metodologia garantiu a empresa estudada uma economia de milhões de dólares além de maior produção, conquistaram a satisfação dos clientes e um maior desempenho dos colaboradores. A ferramenta DMAIC possibilitou maior comunicação e participação em equipe se tornando parte da cultura da empresa, garantindo assim que os colaboradores tivessem o mesmo nível de conhecimento da metodologia e ferramenta auxiliando na comunicação e tomada de decisões da empresa em conjunto com seus funcionários. Utilizando como metodologia de estudo revisão bibliográfica e estudo de caso.
\end{abstract}

Palavras-chave: Seis Sigma. Foco no Cliente. Qualidade. Menor Custo.

\begin{abstract}
The current scenario is increasingly competitive and the most demanding customers, thinking about it began the search for total quality and high competitiveness at the lowest possible cost. Six Sigma, a methodology created in the 1980s, has this objective, it uses the statistical basis to achieve the established goals. It aims to achieve excellence in competitiveness with the continuous improvement of processes, being a managerial strategy to accelerate the improvement in processes, products and services. The expression Sigma measures the capacity of the process to work without fail. The reduction in the variation in the result
\end{abstract}


delivered to customers at a rate of 3.4 imperfections per million or $99.99966 \%$ of perfection is the definition of Six Sigma. The method becomes unique and extremely effective due to its structure and focus on reducing the variability of the process. The study aims to present the concepts, definitions and tools of Six Sigma and understand its effectiveness. The methodology ensured the company studied savings of millions of dollars in addition to greater production, achieved customer satisfaction and increased performance of employees. The DMAIC tool enabled greater communication and team participation, becoming part of the company's culture, thus ensuring that employees had the same level of knowledge of the methodology and tool assisting in the communication and decision-making of the company together with its employees. Using as methodology of study bibliographic review and case study.

Keywords: Six Sigma. Customer Focus. Quality. Lowest Cost.

\section{INTRODUÇÃO}

O avanço da produção e competitividade no mercado obrigou as empresas a se manterem vivas por meio da evolução constante, oferecendo qualidade e os serviços exigidos pelos clientes. Pensando nisso, iniciaram a luta para conquistar a melhoria na produção, buscando, assim, a qualidade total e alta competitividade em relação a seus concorrentes, com menor custo de produção. Com isso surgiram metodologias que visam a esses resultados.

O Seis Sigma, metodologia criada na Motorola em 1987, segundo Werkema (2004), recentemente ganhou mais destaque, e tem como objetivo melhorar a qualidade dos produtos, dispondo de ferramentas com grande embasamento estatístico, com agrupamentos entre si, visando à diminuição de desperdícios no processo.

\footnotetext{
As organizações de sucesso entendem a variabilidade do processo e passam a controlá-la como forma de redução de falhas e aumento da confiabilidade, e isso são muito mais efetivas que simplesmente procurar eliminar os defeitos. Ao trabalhar com a variabilidade, a metodologia Seis Sigma consegue resultados dificilmente atingidos por outras metodologias. Como meio para descrever e controlar a variabilidade, essas organizações usam a estatística de uma forma inteligente, que pode ser resumida da seguinte maneira:

Obtenha os dados de seu processo, transforme em dados estatísticos, resolva o problema estatisticamente, transforme os resultados em dados de seu processo (Mari, 1997 apud ROTONDARO, 2011, p. 14)
}

No Brasil, inúmeras empresas adotaram essa metodologia, em destaque estão: Ambev; Braskem; Celma/GE; Gerdau; Multibrás; Nokia; Tigre Tubos e Conexões; Tupy Fundições e Votorantim Cimentos (RODRIGUES, 2013). 
O tema do presente artigo é "Um estudo sobre a metodologia Seis Sigma", o qual tem o intuito de compreender sua eficácia, bem como apresentar seus conceitos, definições e ferramentas para empresas que desejam alcançar, maximizar e assegurar o sucesso comercial, com base na compreensão das necessidades do cliente. Um conceito focado no cliente e no produto.

O estudo será estruturado nas seguintes sessões: 1 Introdução; 2 O Que é Seis Sigma? 2.1 Seleção de Projetos; 2.2 Organizando a Equipe Seis Sigma; 2.3 O Modelo DMAIC; 2.4 Calculando a Capacidade Sigma; 3 Procedimentos Metodológicos; 4. Apresentação de Caso: Seis Sigma na Industria de Fabricação Elétrica; 4.1. Contextualização 4.2 Infraestrutura Organizacional; 4.3 Desafios Encontrados; 4.4 Benefícios Conquistados; 4.5 Técnicas e Ferramentas Mais Comuns Utilizadas; 4.6 Principais Aprendizados; 5 Considerações Finais.

\section{O QUE É SEIS SIGMA?}

FIGURA 1- ANÁLISE DO PROCESSO

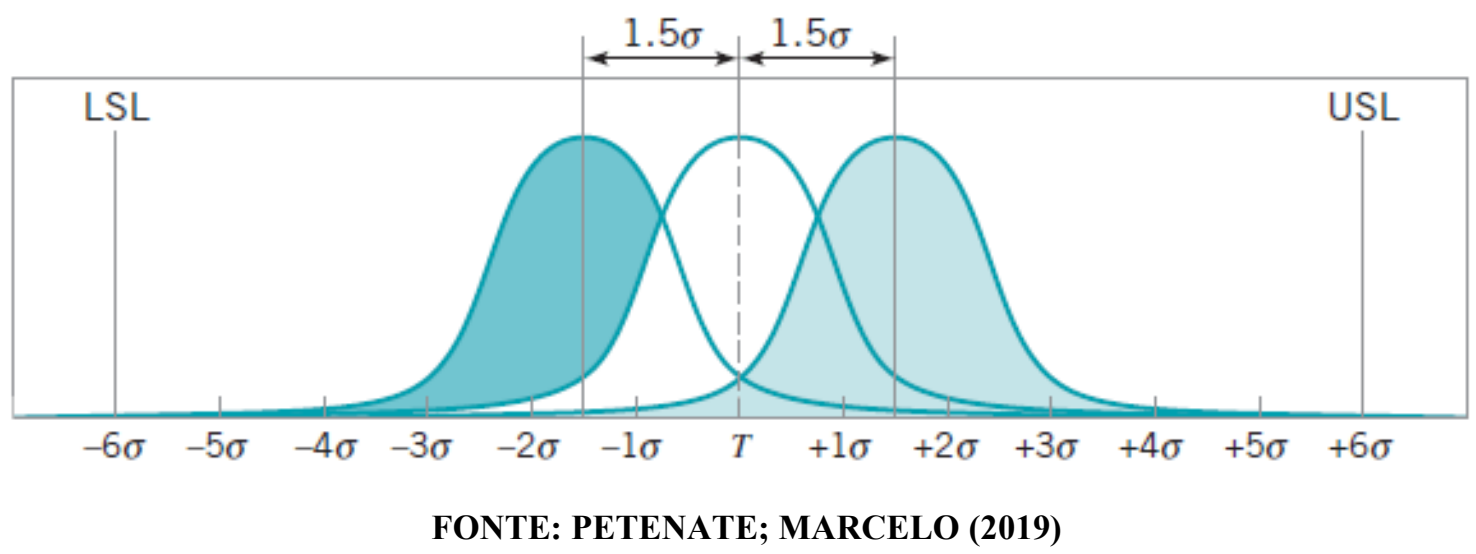

Foi criado pelo engenheiro e cientista Bill Smith, da Motorola, mas foi Bob Galvin, que ocupava o cargo de CEO da companhia, que lançou o "Seis Sigma Quality” na Motorola, com o objetivo de alcançar a qualidade Seis Sigma em 5 anos (DEVANE, 2004).

De acordo com Werkma (2004), foi uma metodologia apresentada visando melhorar a qualidade de seus produtos que na época eram inferiores aos dos concorrentes.

Seis Sigma é a inflexível e rigorosa busca de variação em todos os processos críticos para alcançar melhorias contínuas e quânticas que impactam os índices de uma organização, aumentando a satisfação e a lealdade dos clientes. É uma iniciativa organizacional projetada para criar processos de manufatura, serviços ou administrativo que gere no máximo 3,4 defeitos por milhão de oportunidades. A ferramenta de melhoria empregada na implantação dos projetos Seis Sigma é o 


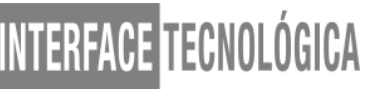

DMAIC, sigla que significa Definir- Medir- Analisar- Implantar- Controlar (RASIS, 2003, p. 127 apud MANI, 2008, p.115).

Para ROTONDARO (2011), é uma metodologia para alcançar, maximizar e manter o sucesso comercial, compreendendo as necessidades do cliente, concentrando-se no cliente e no produto. É voltada para a qualidade, levando em consideração todos os aspectos importantes de um negócio, com o objetivo de conquistar a excelência na melhoria contínua de processos.

Tem como objetivo de atingir a excelência na competitividade com a melhoria contínua dos processos, sendo uma estratégia gerencial para acelerar o aperfeiçoamento em processos, produtos e serviços. A expressão Sigma mensura a capacidade do processo a trabalhar isento de falhas. A contenção da oscilação no resultado cedido aos clientes em uma taxa de 3,4 imperfeições por milhão ou 99,99966\% de perfeição define o Seis Sigma. O método se torna único e extremamente eficaz pela sua estruturação e foco na redução da variabilidade do processo (ROTONDARO, 2011).

\subsection{Seleção de Projetos}

De acordo com o PMBOK (2000), a incitação para o início do projeto pode ser uma exigência ou oportunidade de negócio, causada por uma das seguintes situações:

- Demanda de mercado;

- Necessidade do negócio;

- Um pedido ou exigência do cliente;

- Avanços tecnológicos;

- Exigência legal.

Para uma alocação assegurada, deve-se examinar o que é crítico para a qualidade Critical to Quality (CTQ). Para definir o CTQ tem que se perguntar o que é crítico para o mercado e quais são os processos críticos, buscando diferenciar o que é crítico para a qualidade e para as demandas do mercado, sendo denominada CTQex para a externa e CTQin para as internas como demonstrado na figura 2, depois que se conhece o CTQ o projeto Seis Sigma deve ser aplicado para reduzir a variabilidade dos processos. 
FIGURA 1-ALVO DOS PROJETOS SEIS SIGMA

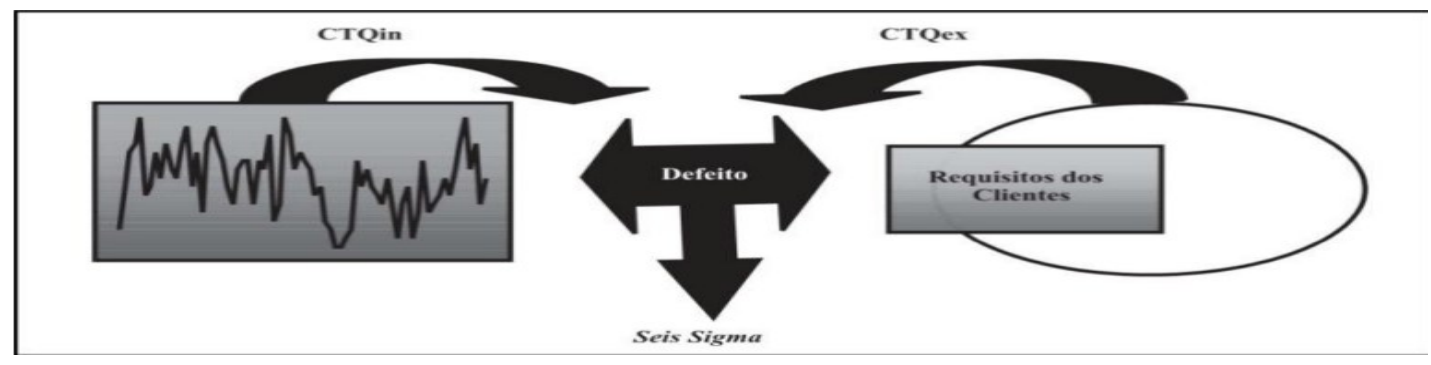

FONTE: CARVALHO (2002)

Farsijani et al. (2015) apresentam uma proposta em que a divisão dos projetos maiores com enfoque na demanda por recursos (tempo e esforço), e que sejam coordenados de modo independente, um a seguir do outro ou conjuntamente.

O estudo realizado por Marzagão e Carvalho (2016) reforça a relevância da diminuição da complexidade, confirmando que quanto mais o projeto é menos complexo seu desempenho apresenta grande melhora.

Segundo Saghaei e Didehkhani (2011, p. 722, apud CONDÉ, 2018, p. 20) “A identificação dos critérios do procedimento de seleção é a parte mais importante e desafiadora de qualquer processo de seleção.”

\subsection{Organizando a Equipe Seis Sigma}

Os projetos de melhoria embasado no método é gerido pelos chamados green belt ou black belt, sendo respectivamente o responsável por projetos de nível intermediário e o responsável por projetos mais complexos, já o sponsor (patrocinador) exerce o papel de dono do negócio, representando a parte estratégica dos projetos para o corpo diretivo, aprovando alterações de layout, liberando verba para financiamentos, entre outros (WERKEMA, 2012).

De acordo com Pyzdek (2003). Os participantes de equipes dos projetos costumam adotar os seguintes papeis:

- Executivo líder: é encarregado pelo desempenho da empresa, ele proporciona os meios para que os objetivos sejam alcançados;

- Champion: uma pessoa que compreende a metodologia Seis Sigma, que desenvolve uma visão holística dentro da empresa, normalmente é ocupada pelo vice-presidente;

- Sponsor: representa a parte estratégica, desenvolve o papel de dono do negócio; 


\section{WNEREFGEETECHOLOGGCA}

- Master black belt: o papel de maior nível em termos de conhecimento técnico, lidera e fornece conhecimento aos black belts. Deve possuir grande conhecimento em estatística e ser hábil para ensinar;

- Black belt: trabalham integralmente na liderança da equipe de projeto, sempre estar envolvido nas mudanças organizacionais e possuir habilidades estatísticas e analíticas;

- Green belt: podem liderar o projeto, só que em um nível de menor complexidade e devem ser auxiliados pelos black belts.

- Yellow belt: capacitados para gerir atividades complexas e delinear melhorias para alcançar os resultados desejados (IPOGblog, 2019).

Observa-se que cada funcionário tem uma função no programa, independente do seu nível hierárquico, integrando assim todos em busca do mesmo objetivo e os mantendo motivados a implantar de forma eficaz a metodologia.

\subsection{O Modelo Definir, Medir, Analisar, Incorporar, Controlar (DMAIC)}

A ferramenta utilizada na metodologia é o DMAIC - Define, Measure, Analyze, Incorparate, Control (definir, medir, analisar, incorporar, controlar) o que baseado nos estudos de Costa Neto; Canuto (2010) é classificado como uma nova roupagem do ciclo PDCA.

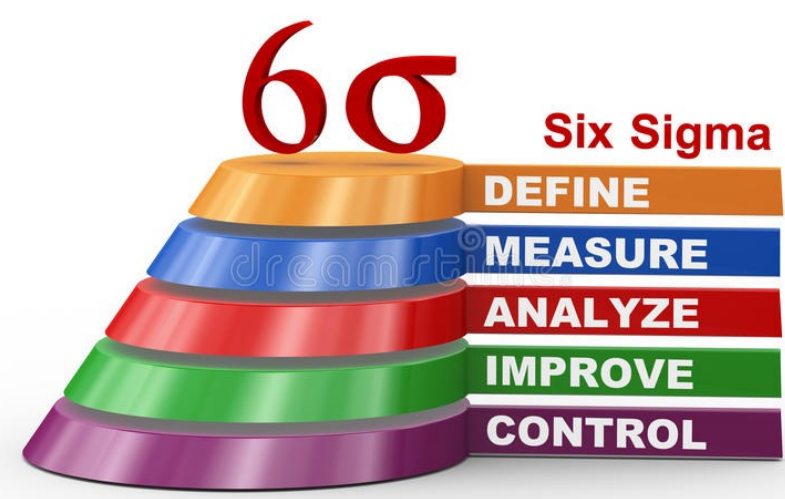

Fonte: Dreamstime (2019) 


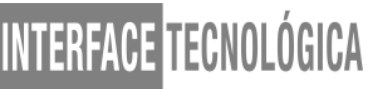

A ferramenta é dividida em cinco etapas, conforme citado por SANTOS (2015):

- D- Define: é a fase em que toda a equipe deve saber perfeitamente o problema a ser resolvido, como será os procedimentos para o desenvolvimento e a alta definição do escopo do projeto, após a equipe estar totalmente estruturada ficam responsáveis em dar início ao projeto definido.

- M- Medir: nesta fase devem-se coletar dados sobre o desempenho atual da área em análise, deve levantar dados que evidenciem as oportunidades de melhoria. A coleta dos dados nessa etapa é essencial para quantificar o problema/oportunidade.

- A- Analyze: os dados coletados passam por uma transformação para se tornarem em informações valiosas, que serão utilizadas para identificação dos problemas. É a etapa em que se analisam as variáveis críticas do processo para determinar as causas de cada problema de acordo com sua prioridade.

- I- Incorparate: com a análise finalizada e os problemas detectados corretamente, nesta fase devem-se planejar e aplicar ações que corrijam os problemas para atingir a melhoria desejada.

- C- Control: para que o programa mantenha seu sucesso conquistado, nesta fase os planos e procedimentos devem garantir controlar e monitorar as melhorias alcançadas para que sejam mantidas em longo prazo, e registrar constantemente as mudanças no processo e se necessário corrigi-las.

Um programa de excelência com o DMAIC, sua implantação só obterá sucesso se for aplicada com planos de ação para melhoria de qualidade, sendo ajustada a estratégica que será seguida (SANTOS, 2010).

\section{PROCEDIMENTOS METODOLÓGICOS}

Para a elaboração deste artigo foi utilizada a metodologia de pesquisa bibliográfica e estudo de caso. Segundo Gil, (2002, p.44) a pesquisa bibliográfica é elaborada com base em material já preparado, formado principalmente de livros, artigos científicos e sites.

O estudo de caso é uma modalidade de pesquisa abundantemente utilizada nas ciências sociais e biomédicas. Consiste no estudo profundo e minucioso de um ou poucos objetos, de maneira que possibilite seu amplo e detalhado conhecimento, tarefa praticamente impossível mediante outros delineamentos já considerados. Consiste em coletar e analisar informações 


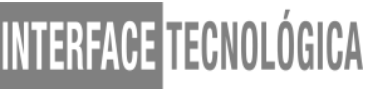

sobre determinado indivíduo, grupo ou comunidade, a fim de estudar aspectos variados que sejam objeto de pesquisa (GIL, 2002, p.54).

O estudo de caso parte de uma situação-problema que se busca assimilar e para isso o pesquisador deve coletar informações sobre o contexto e suas variáveis. Os dados podem ser coletados mediante pesquisas bibliográficas, documentais, pesquisa de campo, ou qualquer método adequado para a coleta de informações. Sendo utilizado nesse estudo a pesquisa bibliográfica.

O estudo de caso em questão segue as seguintes etapas:

1. Identificar um problema de pesquisa

2. Indagação dos dados

3. Exploração do contexto (variáveis)

4. Conclusões sobre o problema

\section{APRESENTAÇÃO DE CASO: SEIS SIGMA NA INDÚSTRIA DE FABRICAÇÃO ELÉTRICA}

\subsection{Contextualização}

Baseado no estudo de SANTOS (2019). A empresa Z está localizada no Norte da Índia, atua como um grande fabricante de componentes elétricos. Com um grupo em cerca de 12.000 colaboradores, sendo uma das principais empresas do setor público na Índia. Fabricando turbinas, transformadores etc., que são utilizados em usinas de energia. Atendem o mercado nacional e internacional, com um grande comprometimento com sua qualidade, tem envolvido todos os colaboradores e supervisores em círculos de qualidade, expandiram a aplicação do Seis Sigma da manufatura pura para suportar suas funções (RH, finanças, compras, manutenção, vendas etc.).

\subsection{Infraestrutura Organizacional}

O método foi aplicado por quase 3 anos. Sua aplicação envolveu em sua equipe de liderança um grupo estratégico sendo eles: diretor executivo, diretor de operações, diretor financeiro, diretor de recursos humanos, diretor de qualidade, chefe de produção, chefe de vendas e marketing e o chefe de atendimento ao cliente. Como o campeão do projeto foi denominado o chefe das funções de negócios, sendo capacitado com um treinamento de 1 dia.

Entorno de 60 projetos foram constatados nos 2 primeiros anos do programa, sendo enviados aos Black Belts (que fizeram um curso intensivo de 4 semanas com uma empresa de 


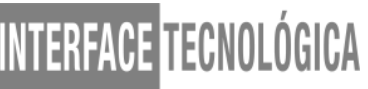

consultoria). A empresa $\mathrm{Z}$ possui atualmente cerca de 5 Master Black Belts, 6 Seis Sigma Champions, 90 Black Belts, 150 Green Belts e mais de 300 Yellow Belts (SANTOS, 2019).

\subsection{Desafios Encontrados}

A empresa se deparou com a dificuldade de manter seus Black Belts no negócio. Após o treinamento e execução dos projetos diversos Black Belts deixaram a empresa Z por empregos nas concorrentes sendo altamente remunerados, criando assim, problemas para encontrar candidatos capacitados para executar o Seis Sigma estrategicamente. Apesar do excelente sistema ERP da empresa, a exatidão dos dados coletados ainda é discutível para os Black Belts (SANTOS, 2019).

\subsection{Benefícios Conquistados}

A empresa $Z$ relatou diversos benefícios dentre eles estão:

- Aumento de mais de 55\% na produtividade de uma máquina de entalhe CNC resultando em uma economia entorno de US \$3.000.000,00 por ano.

- A redução da taxa de retrabalho/rejeição da ovalização do comutador de um motor de tração de $16 \%$ para menos de 3\%. Resultando em uma economia de no mínimo US \$ 2.000.000,00 por ano. Garantindo a redução da espera de montagem e entregas dentro do prazo a seus clientes.

- Foi anexado um hospital com o foco na redução de tempo de espera dos pacientes no ambulatório, resultando em uma redução de $40 \%$, auxiliando para que o atendimento fosse mais rápido e melhor. A satisfação do cliente aumentou em mais de $25 \%$ (SANTOS, 2019).

\subsection{Técnicas e Ferramentas Mais Comuns Utilizadas}

SANTOS (2019) apresenta como as ferramentas e técnicas mais aplicadas para a conquista dos resultados:

- Análise de fornecedor-entrada-processo-saída-cliente (SIPOC)

- Diagrama de Ishikawa (análise de causa e efeito)

Interface Tecnológica - v. 17 n. 2 (2020) 
- Análise de Pareto (análise 80/20)

- Mapeamento de Fluxo de Valor/ Mapeamento de Processos (VSM)

- 8 Formas de análise de desperdícios

- Cartas de controle (gráfico individual, X-bat e R chart, P-chart, U-chart etc.)

- Testes de hipótese (teste $\mathrm{t}$ de 2 amostras, teste F, teste qui-quadrado, teste de KruskalWallis (teste não paramétrico), etc.)

- Análise conjunta-usada para registrar o feedback de clientes em potencial.

\subsection{Principais Resultados e Discussões}

Com o projeto Seis Sigma houve aumento na satisfação do cliente, maior produtividade, redução de custos, melhora da qualidade e maior empenho dos colaboradores.

A empresa $\mathrm{Z}$ passou a tomar decisões eficientes com base em dados. $\mathrm{O}$ uso de DMAIC para solução de problemas otimizou a participação em equipe e a comunicação em várias funções da empresa. Se tornou obrigatório a conscientização sobre a metodologia Seis Sigma e o modelo DMAIC para o método ser de conhecimento de todos da mesma forma.

Perceberam que a metodologia não deveria se limitar a fabricação, expandindo assim para outras áreas da empresa. Foram lançados projetos inovadores em áreas como finanças, recursos humanos etc.

O projeto tem o poder de aumentar a produtividade, satisfação do cliente, reduzir os custos. Seus benefícios são vários se aplicados a metodologia de maneira correta utilizando as ferramentas mais adequadas para sua empresa. À análise de projetos e medidas a serem tomadas tem alta influência nos resultados obtidos e após sua implantação em determinada área seu valor é mais apreciado tendo grandes chances de expandir a metodologia para outros departamentos da organização.

\section{CONSIDERAÇÕES FINAIS}

O estudo evidenciou que mesmo tendo sido criada na década de 80 os diversos benefícios que a metodologia Seis Sigma proporciona são completamente satisfatórios, possui um embasamento estatístico que auxilia na redução de desperdícios e melhora da qualidade 


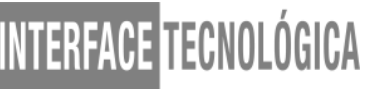

garantindo um ótimo produto com o foco na satisfação do cliente, teve como objetivo apresentar seus conceitos, ferramentas e entender a metodologia sendo aplicada na pratica, o que foi possível graças a revisão bibliográfica e o estudo de caso aqui explorados.

A metodologia garantiu a empresa $\mathrm{Z}$ uma economia de milhões de dólares além de maior produção, conquistaram a satisfação dos clientes e um maior desempenho dos colaboradores.

A ferramenta DMAIC possibilitou maior comunicação e participação em equipe, se tornando parte da cultura da empresa, garantindo assim que os colaboradores tivessem o mesmo nível de conhecimento da metodologia e ferramenta auxiliando na comunicação e tomada de decisões da empresa em conjunto com seus funcionários.

Considerando os resultados obtidos neste trabalho, são feitas as seguintes sugestões para futuras pesquisas: Além do DMAIC outras ferramentas foram utilizadas para o sucesso do método Seis Sigma, com isso ficam como sugestão para estudo (SIPOC, diagrama de Ishikawa, VSM, entre outros).

Outra sugestão seria a metodologia Lean Seis Sigma, por trazer a melhoria contínua e eficiência do Lean para o Seis Sigma obtendo assim o melhor de cada uma das metodologias.

\section{REFERÊNCIAS}

Condé, G. C., \& Martens, M. L. Projetos lean manufacturing para geração de portfólio: uma revisão da literatura. Exacta - EP, São Paulo, v. 16, n. 1, p. 103-122, 2018

COSTA NETO, P. L. D. O.; CANUTO, S. A. Administração com qualidade:

Conhecimentos necessários para a gestão moderna. São Paulo: Bluncher, 2010.

DEVANE, T. Integrating lean six sigma and high performance organizations. San Francisco: Pfeiffer Wiley Imprint, 2004.

FARSIJANI, H.; NIKABAI, M. S.; AMIRIMOGHADAM, H. Six sigma project selections using fuzzy networkanalysis and fuzzy MADM. Decision Science Letters, v 4, p.87-96, 2015.

Gil, A. C. Como elaborar projetos de pesquisa .4 ed. São Paulo: Atlas, 2002. 
MARZAGÃO, D. S. L.; CARVALHO, M. M. Critical success factors for Six Sigma projects. International Journal of Project Management, v. 34, n. 8, p. 1505-1518, 2016.

Modelo DMAIC. Dreamstime, 2019. Disponível em:https://pt.dreamstime.com/imagens-destock-royalty-free-melhoria-de-processo-seis-sigma-image28804859. Acesso em: 27 de nov. de 2019.

PYSDEK, T. The Six Sigma Handbook. McGraw-Hill, e-book edition. New York, 2003.

PROJECT MANAGEMENT INSTITUTE. A Guide to the Project Management Body of Knowledge - PMBOK® Guide 2000 Edition, Pennsylvania-USA 2000

RAMOS, A. et al. Seis Sigma: Estratégia Gerencial para a Melhoria de Processos, Produtos e Serviços. 1. ed. São Paulo: Editora ATLAS S.A., 2013.

RODRIGUES, S. G. Aplicação da Lógica Paraconsistente na seleção de alternativas de Transporte público. Dissertação de Mestrado em Transportes, Publicação T.DM003A/2013, Brasília, 2013. Departamento de Engenharia Civil e Ambiental, Universidade de Brasília.

ROTONDARO, R. Seis Sigma: Estratégia Gerencial para a Melhoria de Processos, Produtos e Serviços. 1. ed. São Paulo: Editora ATLAS S.A., 2011.

Saghaei, A., \& Didehkhani, H. (2011). Developing an integrated model for the evaluation and selection of six sigma projects based on ANFIS and fuzzy goal programming. Expert Systems with Applications, 38(1), 721-728.

SANTOS, V. Estudo de Caso: Seis Sigma na Indústria de Fabricação Elétrica. FM2S, 2019. Disponível em:https://www.fm2s.com.br/estudo-de-caso-seis-sigma-2/. Acesso em: 10 de fev. de 2020.

WERKEMA, M. C. C. Criando a cultura Seis Sigma. Série Seis Sigma. Volume 1. Nova Lima, MG: Werkema Ed., 2004.

WERKEMA, M.C.C. Criando a cultura Lean Seis Sigma. 2 ed. Belo Horizonte: Werkema Editora, 2012. VOITTO TREINAMENTO E DESENVOLVIMENTO. Treinamento de Green Belts em Lean Seis Sigma. Apostila do treinamento, rev. 15, 2014.

Yellow Belt em Six Sigma: o que é, diferença entre os Belts e ferramentas. IPOGblog, 2019. Disponível em:https://blog.ipog.edu.br/engenharia-e-arquitetura/yellow-belt/. Acesso em: 04 de mar. de 2020. 